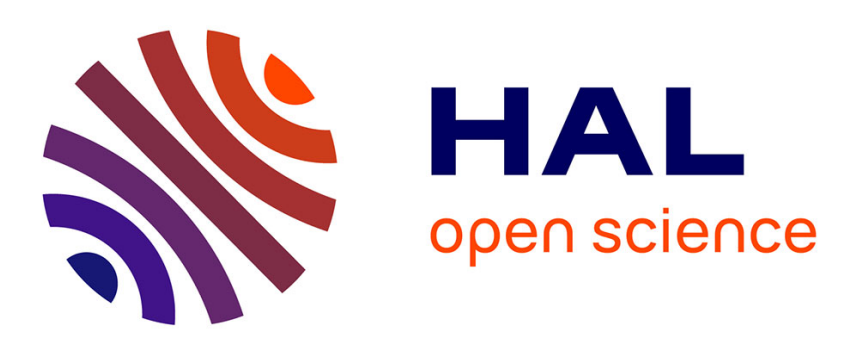

\title{
High-gain observer design for some semilinear reaction-diffusion systems: a transformation-based approach
}

Constantinos Kitsos, Gildas Besancon, Christophe Prieur

\section{To cite this version:}

Constantinos Kitsos, Gildas Besancon, Christophe Prieur. High-gain observer design for some semilinear reaction-diffusion systems: a transformation-based approach. IEEE Control Systems Letters, 2021, 5 (2), pp.629-634. 10.1109/LCSYS.2020.3004742 . hal-02878257

\section{HAL Id: hal-02878257 \\ https://hal.science/hal-02878257}

Submitted on 23 Jun 2020

HAL is a multi-disciplinary open access archive for the deposit and dissemination of scientific research documents, whether they are published or not. The documents may come from teaching and research institutions in France or abroad, or from public or private research centers.
L'archive ouverte pluridisciplinaire HAL, est destinée au dépôt et à la diffusion de documents scientifiques de niveau recherche, publiés ou non, émanant des établissements d'enseignement et de recherche français ou étrangers, des laboratoires publics ou privés. 


\title{
High-gain observer design for some semilinear reaction-diffusion systems: a transformation-based approach
}

\author{
Constantinos Kitsos, Gildas Besançon, and Christophe Prieur
}

\begin{abstract}
The design problem of a high-gain observer is considered for some $2 \times 2$ and $3 \times 3$ semilinear reaction-diffusion systems, with possibly distinct diffusivities, and considering distributed measurement of part of the state. Due to limitations imposed by the parabolic operator, for the design of such an observer, an infinite-dimensional state transformation is first applied to map the system into a more suitable set of partial differential equations. The observer is then proposed including output correction terms and also spatial derivatives of the output of order depending on the number of distinct diffusivities. It ensures arbitrarily fast state estimation in the sup-norm. The result is illustrated with a simulated example of a Lotka-Volterra system.
\end{abstract}

Index Terms - high-gain observers, Lotka-Volterra systems, Lyapunov stability, semilinear parabolic systems.

\section{INTRODUCTION}

$\mathbf{T}$ HE problem of high-gain observer design for nonlinear finite-dimensional systems has been widely considered in the literature (see [19] and references therein). Briefly, it relies on a tuning coefficient (gain), to be chosen large enough so as to ensure arbitrarily fast exponential convergence. In [20], [21], this approach was extended to infinite-dimensional systems, more precisely, hyperbolic systems of balance laws, while utilizing distributed measurement of a part of the state.

Most techniques for the state estimation of linear distributed parameter systems (the so-called late-lumping ones, see [30] for a survey on state estimation) rely on operator and semigroup-theoretic approaches, Lyapunov-based analysis and backstepping, see for instance [12], [13], [28], [14], [17]. The case of state estimation for nonlinear infinte-dimensional systems, which is significantly more complicated, has been addressed in [31], [4], [9], [24], [3], [27], [8] amongst others, but mainly considering the full state vector on the boundaries as measurement, and without the high gain features.

The present paper aims at providing a solution to a highgain observer design problem for some Lotka-Volterra-like semilinear parabolic systems with 2 and 3 states written in cascade form and considering measurement of the first state only. In this framework with a reduced number of observations being available, the solution to an observer problem is technically not obvious, as the system's differential operator imposes

All the authors are with Univ. Grenoble Alpes, CNRS, Grenoble INP $^{\circ}$, GIPSA-lab, 38000 Grenoble, France, (e-mails: \{ konstantinos.kitsos, gildas.besancon, christophe.prieur $\} @$ gipsa-lab.grenoble-inp.fr)

$\circ$ Institute of Engineering Univ. Grenoble Alpes some limitations. More precisely, the distinct diffusivities do not allow the observer design to be directly feasible, as in the finite-dimensional approach. This work extends a recently introduced transformation-based methodology in [20] and [21] for $2 \times 2$ quasilinear and $3 \times 3$ linear hyperbolic systems to cases of $2 \times 2$ and $3 \times 3$ semilinear parabolic systems. The class of systems that we study might describe biological predator-prey models and other population and social dynamics phenomena [7], [2]. Such systems have gained significant interest with respect to controllability and one can refer to [26] (see also [11]). For Lyapunov techniques on parabolic systems, one can refer to [23]. Moreover, observer design for (finitedimensional) Lotka-Volterra systems has been addressed in [5].

The main contribution here is a solution to this high-gain observer design problem with reduced number of observations (only the first state) in the presence of distinct diffusivities of the parabolic operator, and considering also semilinear dynamics. A fundamental idea of this paper is to first perform an appropriate infinite-dimensional and lower triangular transformation to map the considered systems into new sets of PDEs, where the parabolic operator is decomposed into a new one with only one diffusivity and a mapping, including spatial derivations of the measured state in its domain. This methodology imposes stronger regularity of system's solutions and results in requiring, additionally to the output correction terms, the injection of ouput's spatial derivatives in the highgain observer dynamics. Notice that the somehow dual controllability problem for cascade systems with a reduced number of control variables runs deep [10], [1], and stronger regularity of the dynamics is imposed in the corresponding studies. The convergence of the observer is then proven for the sup-norm. Moreover, in the presence of the nonlinearities, we plug in sufficiently smooth saturation functions in the nonlinearities of the proposed observer, while system's solutions remain uniformly bounded with a priori known bounds.

The paper is organized as follows. The sufficient conditions and a solution to the high-gain observer design problem are presented in Section II, where Theorem 1 constitutes the main result. The proof of Theorem 1, containing also the infinitedimensional transformation that we use to obtain a target system for observer design, are presented in Section III. In Section IV, we apply our methodology to a $2 \times 2$ diffusional LotkaVolterra system. Conclusions and perspectives are discussed in Section V.

Notation: For a given $w$ in $\mathbb{R}^{n},|w|$ denotes its usual 
Euclidean norm. For a given matrix $A$ in $\mathbb{R}^{n \times n}, A^{\top}$ denotes its transpose, $|A|:=\sup \{|A w|,|w|=1\}$ is its induced norm and $\operatorname{Sym}(A)=\frac{A+A^{\top}}{2}$ stands for its symmetric part. By $\operatorname{eig}(A)$ we denote the minimum eigenvalue of a symmetric matrix $A$. By $I_{n}$ we denote the identity matrix of dimension $n$. For a continuous $\left(C^{0}\right)$ mapping $[l, L] \ni x \mapsto u(x) \in \mathbb{R}^{n}$ we adopt the notation $\|u\|_{\infty}:=\max \{|u(x)|, x \in[l, L]\}$ (with $\left.\|u\|_{0}:=\|u\|_{\infty}\right)$. For a $q$ - times continuously differentiable $\left(C^{q}\right)$ mapping $[l, L] \ni x \mapsto u(x) \in \mathbb{R}^{n}$ we adopt the notation $\|u\|_{q}:=\sum_{i=0}^{q}\left\|\partial_{x}^{i} u\right\|_{\infty}$. For a differentiable mapping $f$, by $D f$ we denote its Jacobian.

\section{Problem Statement and Main Result}

In this work, being motivated by applications to information diffusion by multiple sources in social media [29], we consider a system of two or three one-dimensional semilinear parabolic equations with Neumann boundary conditions, written in the following form:

$$
\begin{gathered}
u_{t}=D u_{x x}+A\left(u_{1}\right) u+f(u) \text { in }(0,+\infty) \times(l, L) \\
u(0, x)=u^{0}(x), x \in[l, L] \\
u_{x}(t, l)=0, u_{x}(t, L)=0, t \in(0,+\infty) .
\end{gathered}
$$

where $L>l_{1} \geq 0$ define the space domain, and $u=$ $\left(\begin{array}{lll}u_{1} & \cdots & u_{n}\end{array}\right)^{\top}$ is the 2 or 3 -dimensional vector state (namely $n=2$ or $n=3$ ),

$$
\begin{aligned}
& D=\operatorname{diag}\left(d_{1}, \ldots, d_{n}\right) ; d_{i}>0, i=1, \ldots, n, \\
& A\left(u_{1}\right):= \begin{cases}\left(\begin{array}{cc}
0 & a_{12} u_{1} \\
0 & 0
\end{array}\right), & \text { if } n=2, \\
\left(\begin{array}{ccc}
0 & a_{12} & 0 \\
0 & 0 & a_{23} \\
0 & 0 & 0
\end{array}\right)(=: A), & \text { if } n=3,\end{cases} \\
& f(u):=\left\{\begin{array}{cc}
r_{1} u_{1}\left(1-\frac{u_{1}}{K_{1}}\right) \\
r_{2} u_{2}\left(1-\frac{u_{2}}{K_{2}}\right)+a_{21} u_{2} u_{1}
\end{array}\right), \quad \text { if } n=2,
\end{aligned}
$$

for some constants $a_{21}, a_{31}, a_{32}$ and positive constants $a_{12}, a_{23}, r_{i}, K_{i}$. Note here that these two types of systems are henceforth parametrized by $n$, thus, whenever we say $n=2$ or $n=3$, we refer to the above $2 \times 2$ or $3 \times 3$ system, respectively. Assume, also, that initial conditions $u^{0}(\cdot)$, with $u^{0}(\cdot) \geq 0$, belong to the Banach space $\mathcal{X}$, where

$$
\begin{gathered}
\mathcal{X}:=C^{q_{0}}([l, L] ; \mathbb{R}) \times C^{2}\left([l, L] ; \mathbb{R}^{n-1}\right) ; \\
q_{0}:=\max (2,2 q-2) ; \\
q:=\min \left\{i: d_{i}=d_{j}, \forall j=i, i+1, \ldots, n\right\}
\end{gathered}
$$

and $\mathcal{X}$ is equipped with $C^{2}$-norm when $n=2$ and the norm

$$
\|u\|_{\mathcal{X}}:=\left\|u_{1}\right\|_{q_{0}}+\left\|\left(u_{2}, u_{3}\right)\right\|_{2}, \text { when } n=3 \text {. }
$$

From the above definition of $q$, note that $q \in\{1,2,3\}$ (thus $q_{0} \in\{2,4\}$ ) and when diffusivities $d_{i}$ are distinct, we get $q=n$, while $q=1$, when all diffusivities are equal.
We consider a distributed measurement of the first state, written as follows:

$$
y(t, x)=C u(t, x),
$$

where $C=\left(\begin{array}{ll}1 & 0\end{array}\right)$, if $n=2$, or $C=\left(\begin{array}{lll}1 & 0 & 0\end{array}\right)$, if $n=3$.

Remark 1: The above-considered system for $n=2$ is a diffusional Lotka-Volterra system. We underline that for the $3 \times 3$ system, we consider some simpler dynamics with respect to $A(\cdot)$, being constant in this case, and, thus, not satisfying the exact form of classical $3 \times 3$ Lotka-Volterra systems in cascade form. An extension of the method we adopt here to general diffusional Lotka-Volterra cascade systems of $n$ species is difficult due to the nonlinearities, and the difficulty increases with the number of distinct diffusivities. Notice that similar difficulties, which increase with the number of transport speeds and the types of nonlinearities, appear in the study of controllability for cascade hyperbolic systems with reduced number of controls [1] and come from the notion of algebraic solvability. For such problems, stronger regularity of the solutions is imposed; in a similar way stronger regularity conditions are assumed here as well, in order to solve the problem of observer design with reduced number of observations.

In [25] it is shown that systems of the form (1), with the present regularity conditions, admit unique local solutions in the classical sense defined therein (see Lemma 1.1) and their regularity can be understood in the usual sense, since the nonlinear source term $A\left(u_{1}\right) u+f(u)$ is regular enough. Particularly, there exist $T>0$ and a unique solution $u$ to (1) on $[0, T)$, with $u \in C^{1}\left([0, T) \times[l, L] ; \mathbb{R}^{n}\right)$ and $u(t, \cdot) \in C^{2}\left([l, L] ; \mathbb{R}^{n}\right), \forall t \in[0, T)$. Furthermore, "quasipositivity" (see [25]) of the nonlinearities implies that these local solutions are non-negative for non-negative initial conditions $u^{0}$. Here, for observer design issues, we introduce an assumption on global existence of nonegative solutions which are also uniformly bounded, assuming additionally some extra regularity. In addition, we assume that, for the case $n=2$, there exists a lower positive bound for system's first state. The latter presents an analogy to the observer design conditions for finite-dimensional systems, see [16].

Assumption 1: System (1) with initial condition $u^{0} \quad$ satisfying compatibility conditions for the space $\mathcal{X}$, admits a unique and uniformly bounded solution $u$ in $C^{\max \left(1, q_{0}-2\right)}([0,+\infty) \times[l, L] ; \mathbb{R}) \times$ $C^{1}\left([0,+\infty) \times[l, L] ; \mathbb{R}^{n-1}\right)$, with $u(t, \cdot) \in \mathcal{X}$, for all $t \in[0,+\infty)$. For $n=3$, considering non-negative initial condition $u^{0}$, this solution remains non-negative. In the case where $n=2$, we have $u^{0}(\cdot)>0$ and its corresponding solution $u_{1}$ satisfies $\inf _{(t, x) \in[0,+\infty) \times[l, L]} u_{1}(t, x)>0$.

Remark 2: It is not unusual to consider global solutions for such systems, by showing that solutions might not blow up on some maximal time interval of existence (see assertions in [25]). Following Theorem 3.5 in [25], we can deduce that a sufficient condition for global existence when $n=3$ is $a_{21}, a_{31}, a_{32} \leq 0$. The stronger regularity that we impose on the solutions can be seen as a consequence of the regularity of the initial conditions and the nonlinear source. The method 
to prove this by finding a priori estimates is standard, see for instance [22, Chap. V] and [15, Chap. 8.3.2].

We are in a position to propose an indirect observer design, as explained below, which deals with the problem of the presence of distinct diffusivities. Let us first consider a symmetric and positive definite matrix $P$ satisfying a Lyapunov equation of the following form for $(t, x) \in[0,+\infty) \times[l, L]$ :

$$
\operatorname{Sym}(P A(y(t, x)))-C^{\top} C \leq-\frac{\eta}{2} I_{n},
$$

for some constant $\eta>0$. Such an inequality is always feasible for $A(y)$ and $C$ satisfying particular structures as the ones we already assumed. More explicitly, for systems with two states $(n=2)$, feasibility of such an inequality requires additionally $y=u_{1}$ to satisfy Assumption 1 (having upper and lower bounds). The existence of such a $P$ satisfying (4) for $\eta>0$ is shown in [16]. When $n=3$, such an inequality is feasible, due to the observability of the pair $A, C$. Furthermore, let us note that such a $P$ is never diagonal. In a possible direct observer design, its asymptotic convergence would be proven by choosing $P$ as a Lyapunov matrix, simultaneously commuting with the diffusion matrix $D$. This commutation property would allow the integration by parts in the Lyapunov analysis and can be only satisfied when $D$ is a scalar matrix, which is not the general case here. For this reason, we propose a transformation into a new system where the parabolic operator is decomposed into a sum of a) a new differential operator, satisfying the previously mentioned commutation, b) a differential operator (and possibly nonlinear) including only the first measured state in its domain and c) a bilinear mapping between a function of the unmeasured state and a differential operator, including only the first state in its domain. Moreover, this transformation is assumed to preserve the triangular structure of $A$ and $f$. That kind of transformation is, therefore, infinitedimensional and lower triangular. More precisely, we show the existence of a linear bounded injective transformation $\mathcal{T}:\left(\mathcal{X},\|\cdot\|_{\mathcal{X}}\right) \rightarrow\left(\mathcal{X},\|\cdot\|_{\mathcal{X}}\right)$, with bounded inverse, which maps initial system into a target system $v$, as follows:

$$
\begin{aligned}
& v=\mathcal{T} u \\
& v_{1}=u_{1} .
\end{aligned}
$$

Such a transformation exists for the considered systems, as shown in the sequel, although existence of a transformation (possibly nonlinear) for more general $n \times n$ Lotka-Volterra systems with distinct diffusivities remains open (see Remark 1). The nature of this transformation indicates the need for stronger regularity of system's solutions as in Assumption 1 and this requirement is linked to the sufficient conditions for controllability problems for cascade systems with reduced number of controls [1] (see also Remark 1).

The target system (T) of PDEs, which is suitable for observer design, satisfies the following equations in $(0,+\infty) \times$ $(l, L)$ :

$$
(\mathrm{T})\left\{\begin{array}{l}
v_{t}(t, x)=d_{n} v_{x x}(t, x)+A\left(v_{1}(t, x)\right) v(t, x) \\
+f(v(t, x))+\mathcal{M}_{1}\left[v_{1}(t)\right](x)+\mathcal{M}_{2}\left[v_{1}(t)\right](x) v(t, x) \\
v_{x}(l)=\mathcal{K} v_{1}(l), v_{x}(L)=\mathcal{K} v_{1}(L) \\
y_{v}(t, x)=y(t, x)=C v(t, x)
\end{array}\right.
$$

with initial condition $v(0, x)=v^{0}(x)=\mathcal{T} u^{0}(x)$, where $\mathcal{M}_{1}$ : $C^{q_{0}}([l, L] ; \mathbb{R}) \rightarrow C^{0}\left([l, L] ; \mathbb{R}^{n}\right), \mathcal{M}_{2}: C^{q_{0}}([l, L] ; \mathbb{R}) \rightarrow$ $C^{\max (0,2 q-4)}\left([l, L] ; \mathbb{R}^{n \times n}\right), \mathcal{K}: C^{q_{0}}([l, L] ; \mathbb{R}) \rightarrow \mathbb{R}^{n}$ are nonlinear differential operators acting on $v_{1}$, to be determined in the sequel, depending on the choice of $\mathcal{T}$, and $y_{v}$ is target system's output, which remains equal to original system's output $y$. The existence of such a transformation $\mathcal{T}$ is shown in Section III below.

We are now in a position to propose a high-gain observer for target system (T) satisfying the following equations in $(0,+\infty) \times(l, L):$

$$
\begin{aligned}
& \hat{v}_{t}(t, x)=d_{n} \hat{v}_{x x}(t, x)+A(y(t, x)) \hat{v}(t, x) \\
& +\Theta P^{-1} C^{\top}(y(t, x)-C \hat{v}(t, x))+\left(f \circ s_{\delta}\right)(\hat{v}(t, x)) \\
& +\mathcal{M}_{1}[y(t)](x)+\mathcal{M}_{2}[y(t)](x) \hat{v}(t, x), \\
& \hat{v}_{x}(l)=\mathcal{K} y(l), \hat{v}_{x}(L)=\mathcal{K} y(L),
\end{aligned}
$$

with initial condition $\hat{v}^{0}(x):=\hat{v}(0, x)$ (for some function $\hat{v}^{0}$ in $\mathcal{X}$, satisfying compatibility conditions for this space). Also, $\Theta:=\operatorname{diag}\left\{\theta, \theta^{2}, \ldots, \theta^{n}\right\}$, with $\theta>1$ being the candidate high-gain constant of the observer, to be selected sufficiently large and precisely determined in the sequel, and $P$ is symmetric and positive definite, satisfying (4) for some $\eta>0$. We also injected in the nonlinear dynamics a vectorvalued function $\mathbb{R}^{n} \ni \hat{v} \mapsto s_{\delta}(\hat{v})=\left(s_{\delta, 1}\left(\hat{v}_{1}\right), \ldots, s_{\delta, n}\left(\hat{v}_{n}\right)\right)$, parametrized by

$$
\delta:=\sup _{t \in[0,+\infty)}\|v(t, \cdot)\|_{\mathcal{X}},
$$

which is a well-defined constant by Assumption 1, in conjunction with (5) and boundedness of $\mathcal{T}$. There is no need, however, to inject this function in the appearing linear terms, since they are already globally Lipschitz, although this is not explicitly written here. Alternatively, one can avoid injecting $s_{\delta}\left(\hat{v}_{1}\right)$ in observer's nonlinear dynamics, but use $y$ instead, and we adopt this simplification in the example of the last section. We assume that $s_{\delta}$ is of class $C^{2}$ and satisfies the following properties:

1) For every $\delta>0$ and $w, \hat{w}$ in $\mathbb{R}$, such that $|w| \leq \delta$, there exists $\omega_{\delta}>0$, such that the following inequality is satisfied:

$$
\left|s_{\delta, i}(\hat{w})-w\right| \leq \omega_{\delta}|\hat{w}-w|, i=1, \ldots, n .
$$

2) There exists $m_{\delta}>0$, such that for every $\hat{w}$ in $\mathbb{R}$,

$$
\left|\frac{d^{j}}{d \hat{w}^{j}} s_{\delta, i}(\hat{w})\right| \leq m_{\delta}, j=0,1,2, i=1, \ldots, n .
$$

We are now in a position to present our main result on the convergence of the proposed high-gain observer.

Theorem 1: Consider system (1) with output (3) and suppose that Assumption 1 holds. Then, there exists a linear bounded injective operator $\mathcal{T}$ with bounded inverse, transforming system (1) into system (T). Let also $P$ be a symmetric and positive definite matrix, satisfying (4) for some $\eta>0$. Then, for $\theta$ large enough, $\mathcal{T}^{-1} \hat{v}$ provides an estimate for the solution $u$ to (1), where $\hat{v}$ is the unique solution to observer system (7). More precisely, for every $\kappa>0$, there exists $\theta_{0} \geq 1$, such that for every $\theta \geq \theta_{0}$, the following holds for all $t \geq 0$ :

$$
\left\|\mathcal{T}^{-1} \hat{v}(t, \cdot)-u(t, \cdot)\right\|_{\infty} \leq c e^{-\kappa t}\left\|\mathcal{T}^{-1} \hat{v}^{0}(\cdot)-u^{0}(\cdot)\right\|_{\mathcal{X}},
$$


for some $c>0$, polynomial in $\theta$.

\section{Observer Convergence Proof}

In this section, we prove Theorem 1.

First, we show the existence of $\mathcal{T}$ of the form (5) mapping (1)-(3) into target system (T). Let us choose

$$
\mathcal{T}:= \begin{cases}I_{2}, & n=2, \\
\left(\begin{array}{ccc}
1 & 0 & 0 \\
b \partial_{x}^{2} & 1 & 0 \\
0 & 0 & 1
\end{array}\right), & n=3\end{cases}
$$

where $b:=\frac{d_{3}-d_{2}}{a_{12}}$, with $\mathcal{T}$ being obviously invertible. Then, applying this transformation to the initial system, we obtain system (T) with

$$
\begin{aligned}
& \mathcal{M}_{1}\left[v_{1}\right]:=\left\{\begin{array}{cc}
\left(\begin{array}{c}
\left(d_{1}-d_{3}\right) \partial_{x}^{2} v_{1} \\
0
\end{array}\right), & n=2, \\
\left(d_{1}-d_{3}-a_{12} b\right) \partial_{x}^{2} v_{1} \\
\left(\begin{array}{c}
{\left[b\left(d_{1}-d_{3}\right) \partial_{x}^{4}+b\left(r_{1}-r_{2}\right) \partial_{x}^{2}\right] v_{1}-} \\
-b\left(a_{21}+2 \frac{r_{1}}{K_{1}}\right) u_{1} \partial_{x}^{2} v_{1}- \\
-2 b \frac{r_{1}}{K_{1}}\left(\partial_{x} v_{1}\right)^{2}-b^{2} \frac{r_{2}}{K_{2}}\left(\partial_{x}^{2} v_{1}\right)^{2} \\
0
\end{array}\right), & n=3,
\end{array}\right. \\
& \mathcal{M}_{2}\left[v_{1}\right]:=\left\{\begin{array}{ccc}
0, & & n=2, \\
\left(\begin{array}{ccc}
0 & 0 & 0 \\
0 & 2 b \frac{r_{2}}{K_{2}} \partial_{x}^{2} v_{1} & 0 \\
0 & 0 & -b a_{32} \partial_{x}^{2} v_{1}
\end{array}\right), & n=3,
\end{array}\right. \\
& \mathcal{K}:=0 \text {, if } n=2, \\
& \mathcal{K}:=\left(\begin{array}{c}
0 \\
b \partial_{x}^{2} \\
0
\end{array}\right) \text {, if } n=3 \text {. }
\end{aligned}
$$

We note here some properties, that are invoked later in the well-posedness of the observer and its convergence proof. First, notice that by virtue of Assumption 1 on boundedness of the system's solutions in $\|\cdot\|_{\mathcal{X}}$, boundedness of mapping $\mathcal{T}$, and the dynamics of target system $(\mathrm{T}), v_{t}, v_{x x}$, and $v_{t x x}$ are uniformly bounded in the sup-norm. Now, due to continuity of the nonlinear operators $\mathcal{M}_{1}, \mathcal{M}_{2}$, we get

$$
\begin{gathered}
\sup _{\|y\|_{q_{0}} \leq \delta}\left|\mathcal{M}_{1}[y]\right|<+\infty \\
\sigma_{\delta}:=\sup _{\|y\|_{q_{0}} \leq \delta}\left|\mathcal{M}_{2}[y]\right|, \sigma_{\delta}^{\prime}:=\sup _{\|v\|_{\mathcal{X} \leq \delta}}\left|\mathcal{M}_{2}\left[C v_{t}\right]\right|<+\infty
\end{gathered}
$$

Furthermore, in view of boundedness of solutions (Assumption 1 ), dynamics of $v$, and properties (8), for any $\delta>0$, there exist constants $L_{\delta}, L_{\delta}^{\prime}>0$, such that for every $v, \hat{v}$ in $\mathcal{X}$, with $\|v\|_{\mathcal{X}} \leq \delta$, the following inequalities are satisfied for all $x$ in $[l, L]:$

$$
\begin{aligned}
& \left|\left(f \circ s_{\delta}\right)(\hat{v}(x))-f(v(x))\right| \leq L_{\delta}|\hat{v}(x)-v(x)|, \\
& \left|D\left(f \circ s_{\delta}\right)(\hat{v}(x)) \cdot \hat{v}_{t}(x)-D f(v(x)) \cdot v_{t}(x)\right| \\
& \leq L_{\delta}^{\prime}\left(|\hat{v}(x)-v(x)|+\left|\hat{v}_{t}(x)-v_{t}(x)\right|\right) .
\end{aligned}
$$

Now, to show well-posedness of the observer system, we recall general existence results for systems written in the form $\dot{z}(t)=\mathcal{A} z(t)+F(y(t), z(t))$, see for instance [6] (see also [18, Example 3.6, p. 75]). Here $\mathcal{A}$ is the parabolic operator with domain $\mathcal{D}(\mathcal{A})=\left\{z \in C^{2}\left([l, L] ; \mathbb{R}^{n}\right), z^{\prime}(l)=\mathcal{K} y(l), z^{\prime}(L)=\mathcal{K} y(L)\right\}$ and $F(y(t), z(t)) \quad:=A(y(t)) z(t)+\mathcal{M}_{2}[y(t)] z(t)+$ $\left(f \circ s_{\delta}\right)(z(t))+\Theta P^{-1} C^{\top}(y(t)-C z(t))+\mathcal{M}_{1}[y(t)]$ is the nonlinear source term of the observer, which is uniformly Lipshitz continuous with respect to $z$ when system's output $y$ satisfies Assumption 1. Then, the existence of a unique local solution $z$ in $C^{1}\left(\left[0, T_{1}\right) \times[l, L] ; \mathbb{R}^{n}\right)$ with $z(t) \in$ $C^{2}\left([l, L] ; \mathbb{R}^{n}\right), \forall t \in\left[0, T_{1}\right)$ (for some $T_{1}>0$ ) is guaranteed. Following the continuous differentiability of the nonlinear source, existence in space $\mathcal{X}$ for $n=q=3$ can be deduced by considering the initial value problem for $\dot{z}_{1}$, which can be written as $\frac{d}{d t} \dot{z}_{1}(t)=\mathcal{A}_{1} \dot{z}_{1}(t)+$ $G(t, y(t), \dot{y}(t), z(t), \dot{z}(t)) ; \mathcal{A}_{1} w:=d_{n} w^{\prime \prime}$, with $\mathcal{D}\left(\mathcal{A}_{1}\right)=$ $\left\{\dot{z}_{1} \in C^{2}((l, L) ; \mathbb{R}), \dot{z}_{1}^{\prime}(l)=C \mathcal{K} \dot{y}(l), \dot{z}_{1}^{\prime}(L)\right.$

$=C \mathcal{K} \dot{y}(L)\}$ and initial conditions $\dot{z}_{1}(0)$ satisfy compatibility conditions of order $1 . G$ is uniformly Lipschitz with respect to $\dot{z}$, when $y$ satisfies Assumption 1 and, thus, system admits a unique solution $\dot{z}_{1}$ on $\left[0, T_{2}\right)$ for some $T_{2}>0$, belonging to $C^{1}\left(\left[0, T_{2}\right) \times[l, L] ; \mathbb{R}\right)$ with $\dot{z}_{1}(t) \in C^{2}([l, L] ; \mathbb{R}), \forall t \in\left[0, T_{2}\right)$. Concluding, observer system admits a unique solution on $\left[0, T^{*}\right)$, with $T^{*}:=$ $\min \left(T_{1}, T_{2}\right)$, belonging to $C^{\max \left(1, q_{0}-2\right)}\left(\left[0, T^{*}\right) \times[l, L] ; \mathbb{R}\right) \times$ $C^{1}\left(\left[0, T^{*}\right) \times[l, L] ; \mathbb{R}^{n-1}\right)$, with $\hat{u}(t, \cdot) \in \mathcal{X}$, for all $t \in$ $\left[0, T^{*}\right)$.

Let us now proceed to the stability proof. We define a scaled observer error $\varepsilon:=\Theta^{-1}(\hat{v}-v)$, for which we derive the following parabolic equations in $(0,+\infty) \times(l, L)$ :

$$
\begin{gathered}
\varepsilon_{t}(t, x)=d_{n} \varepsilon_{x x}(t, x)+\theta\left(A(y(t, x))-P^{-1} C^{\top} C\right) \varepsilon(t, x) \\
+\mathcal{M}_{2}[y(t)](x) \varepsilon(t, x)+\Theta^{-1}\left(\left(f \circ s_{\delta}\right)(\hat{v}(x))-f(u(x))\right) \\
\varepsilon_{x}(l)=\varepsilon_{x}(L)=0 .
\end{gathered}
$$

Furthermore, for systems with $n=q=3$, by temporarily assuming some extra regularity for $v_{2}, \hat{v}_{2}, v_{3}, \hat{v}_{3}$, which will be dropped by density arguments, we get the following parabolic equations for $\varepsilon_{t}$ :

$$
\begin{gathered}
\varepsilon_{t t}(t, x)=d_{n} \varepsilon_{t x x}(t, x)+\theta\left(A-P^{-1} C^{\top} C\right) \varepsilon_{t}(t, x) \\
+\mathcal{M}_{2}\left[y_{t}(t)\right](x) \varepsilon(t, x)+\mathcal{M}_{2}[y(t)](x) \varepsilon_{t}(t, x) \\
+\Theta^{-1}\left(D\left(f \circ s_{\delta}\right)(\hat{v}(x)) \cdot \hat{v}_{t}(x)-D f(v(x)) \cdot v_{t}(x)\right), \\
\varepsilon_{t x}(l)=\varepsilon_{t x}(L)=0 .
\end{gathered}
$$

To prove the error's exponential stability with respect to its origin, we adopt a Lyapunov-based approach. Let us define a Lyapunov functional $\mathcal{W}_{p}: \mathcal{X} \rightarrow \mathbb{R}$ by

$$
\begin{aligned}
\mathcal{W}_{p}[\varepsilon] & :=\left(\int_{l}^{L} \mathcal{G}_{p}[\varepsilon](x) \mathrm{d} x\right)^{1 / p} ; \\
\mathcal{G}_{p}[\varepsilon](x) & :=\left(\sum_{i=0}^{\bar{q}} \partial_{t}^{i} \varepsilon^{\top}(x) P \partial_{t}^{i} \varepsilon(x)\right)^{p},
\end{aligned}
$$

with $\bar{q}:=\frac{(q-1)(q-2)}{2}$ and $p$ in $\mathbb{N}$. Denoting $W_{p}(t):=$ $\mathcal{W}_{p}[\varepsilon(t)], G_{p}(t):=\mathcal{G}_{p}[\varepsilon(t)], t \in\left[0, T^{*}\right]$, we calculate the 
time-derivative $\dot{W}_{p}$ along the solutions $\varepsilon, \varepsilon_{t}$ to (12), (13) as follows:

$$
\begin{aligned}
\dot{W}_{p} & =\frac{1}{p} W_{p}^{1-p} \int_{l}^{L} p G_{p-1}(x)\left(\varepsilon_{t}(x)^{\top} P \varepsilon(x)\right. \\
& +\varepsilon(x)^{\top} P \varepsilon_{t}(x)+\bar{q} \varepsilon_{t t}(x)^{\top} P \varepsilon_{t}(x) \\
& \left.+\bar{q} \varepsilon_{t}(x)^{\top} P \varepsilon_{t t}(x)\right) \mathrm{d} x .
\end{aligned}
$$

After substituting (12), (13) and performing an integration by parts, $\dot{W}_{p}$ can be written as follows:

$$
\dot{W}_{p}=W_{p}^{1-p}\left(\frac{1}{p} T_{1, p}+T_{2, p}+T_{3, p}\right),
$$

where

$$
\begin{aligned}
& T_{1, p}:=d_{n}^{p}\left[\partial_{x} G_{p}\right]_{l}^{L}, \\
& T_{2, p}:=2 \int_{l}^{L} G_{p-1}\left[\varepsilon^{\top} P \Theta^{-1}\left(\left(f \circ s_{\delta}\right)(\hat{v})-f(u)\right)\right. \\
& +\bar{q} \varepsilon_{t}^{\top} P \Theta^{-1}\left(D\left(f \circ s_{\delta}\right)(\hat{v}) \hat{v}_{t}-D f(v) v_{t}\right) \\
& +\varepsilon^{\top} \operatorname{Sym}\left(P \mathcal{M}_{2}[y]\right) \varepsilon+\bar{q} \varepsilon_{t}^{\top} \operatorname{Sym}\left(P \mathcal{M}_{2}[y]\right) \varepsilon_{t} \\
& +\frac{\bar{q}}{2} \varepsilon_{t}^{\top} P \mathcal{M}_{2}\left[y_{t}\right] \varepsilon+\frac{\bar{q}}{2} \varepsilon^{\top} \mathcal{M}_{2}\left[y_{t}\right] P \varepsilon_{t}-\varepsilon_{x}^{\top} P \varepsilon_{x} \\
& \left.-\bar{q} \varepsilon_{t x}^{\top} P \varepsilon_{t x}\right] \mathrm{d} x, \\
& T_{3, p}:=2 \theta \int_{l}^{L} G_{p-1}\left(\varepsilon^{\top}\left[\operatorname{Sym}(P A(y))-C^{\top} C\right] \varepsilon\right. \\
& \left.+\bar{q} \varepsilon_{t}^{\top}\left[\operatorname{Sym}(P A)-C^{\top} C\right] \varepsilon_{t}\right) \mathrm{d} x .
\end{aligned}
$$

By use of boundary conditions (12b), (13b) and also (4), (10), and (11a), we get

$$
T_{1, p}=0, T_{2, p} \leq \sigma W_{p}^{p}, T_{3, p} \leq-\theta \frac{\eta}{|P|} W_{p}^{p},
$$

with $\sigma:=2 \underline{\underline{\operatorname{eig}}(P)}\left(\sqrt{n}\left(L_{\delta}+\bar{q} L_{\delta}^{\prime}\right)+\sigma_{\delta}(\bar{q}+1)+\bar{q} \sigma_{\delta}^{\prime}\right)$. Now, selecting high-gain $\theta>\max \left(1, \frac{|P|}{\eta} \sigma\right)$, we get by (15) that there exists a $\kappa>0$, such that $\dot{W}_{p}(t) \leq-2 \kappa W_{p}(t), t \in$ $\left[0, T^{*}\right]$. By comparison lemma, we get the following estimate

$$
W_{p}(t) \leq e^{-2 \kappa t} W_{p}(0), t \in\left[0, T^{*}\right] .
$$

By this estimate, we deduce that solutions in $\mathcal{X}$ to the observer system exist globally in time and, therefore, stability result holds for all $t \geq 0$. We invoke, next, the property

$$
\lim _{p \rightarrow \infty} W_{p}=\sum_{i=0}^{\bar{q}}\left\|\partial_{t}^{i} \varepsilon^{\top}(\cdot) P \partial_{t}^{i} \varepsilon(\cdot)\right\|_{\infty},
$$

holding for continuous $\varepsilon, \varepsilon_{t}$ and also the following inequality which is derived by error equations (12):

$$
a_{1}\left(\left\|\varepsilon_{x x}\right\|_{\infty}-\|\varepsilon\|_{\infty}\right) \leq\left\|\varepsilon_{t}\right\|_{\infty} \leq a_{2}\left(\left\|\varepsilon_{x x}\right\|_{\infty}+\|\varepsilon\|_{\infty}\right),
$$

for some constants $a_{1}, a_{2}>0$ that we can easily calculate. Following the above arguments, we can calculate a positive $\bar{c}$ (polynomial in $\theta$ ), for which the following inequality holds:

$$
\begin{aligned}
& \sum_{i=0}^{\bar{q}}\left\|\partial_{x}^{2 i} \hat{v}(t, \cdot)-\partial_{x}^{2 i} v(t, \cdot)\right\|_{\infty} \leq \bar{c} e^{-\kappa t} \\
& \times \sum_{i=0}^{\bar{q}}\left\|\partial_{x}^{2 i} \hat{v}^{0}(\cdot)-\partial_{x}^{2 i} v^{0}(\cdot)\right\|_{\infty}, t \geq 0 .
\end{aligned}
$$

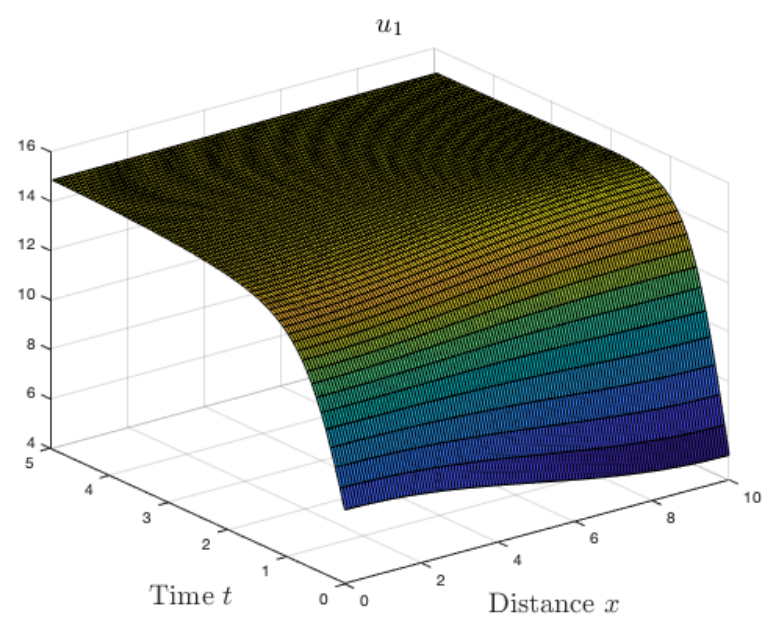

Fig. 1. Time and space evolution of system's output

Now, from continuous embedding of $\mathcal{X}$ in $C^{2}\left([l, L] ; \mathbb{R}^{n}\right)$ and boundedness of $\mathcal{T}$, from the continuous embedding of $C^{2}\left([l, L] ; \mathbb{R}^{n}\right)$ in $C^{0}\left([l, L] ; \mathbb{R}^{n}\right)$ and from boundedness of the continuous extension of the inverse $\mathcal{T}^{-1}$ on $C^{0}\left([l, L] ; \mathbb{R}^{n}\right)$, we deduce stability inequality (9).

The proof of Theorem 1 is complete.

\section{Simulation}

In this section we apply the proposed high-gain observer design to a $2 \times 2$ Lotka-Volterra system (1) as an illustration, with $l=0, L=10$, diffusivities $d_{1}=2, d_{2}=1$ and $a_{12}=$ $0.2, a_{21}=-0.2, K_{1}=15, K_{2}=0.1, r_{1}=0.5, r_{2}=0.01$. We choose initial conditions $u_{1}^{0}(x)=\cos (\pi x / 10)+6, u_{1}^{0}(x)=$ $-3 \cos (\pi x / 10)+9$, such that Assumption 1 is satisfied with a global bound for the solution, which can be known a priori, to be $\delta=20$. The corresponding output is represented in Figure 1 .

The proposed high-gain observer has the form (7). Exploiting the a priori known bounds of system's output, we choose $\eta=0.5, P=\left(\begin{array}{cc}13.4 & 0.667 \\ 0.667 & 0.14\end{array}\right)$, for which (4) holds. We next apply Theorem 1, with observer given by (7) and $\theta=4$. As expected, the convergence of observer state to the unknown state $u$ is guaranteed.

In figures 2, 3 we see the observation errors for each of the states $u_{1}, u_{2}$, after choosing arbitrary observer's initial conditions, satisfying also observer's boundary conditions.

\section{Conclusion}

In this contribution, a high-gain observer for a class of $2 \times 2$ and $3 \times 3$ observable semilinear parabolic systems of Lotka-Volterra type, with possibly distinct diffusivities, has been presented, considering distributed measurement of part of the state. This result constitutes an extension of the highgain observer design for finite-dimensional systems to a class of nonlinear parabolic systems via an indirect approach and, also, an extension of previous works towards this direction for hyperbolic systems. To overcome a technical obstacle 


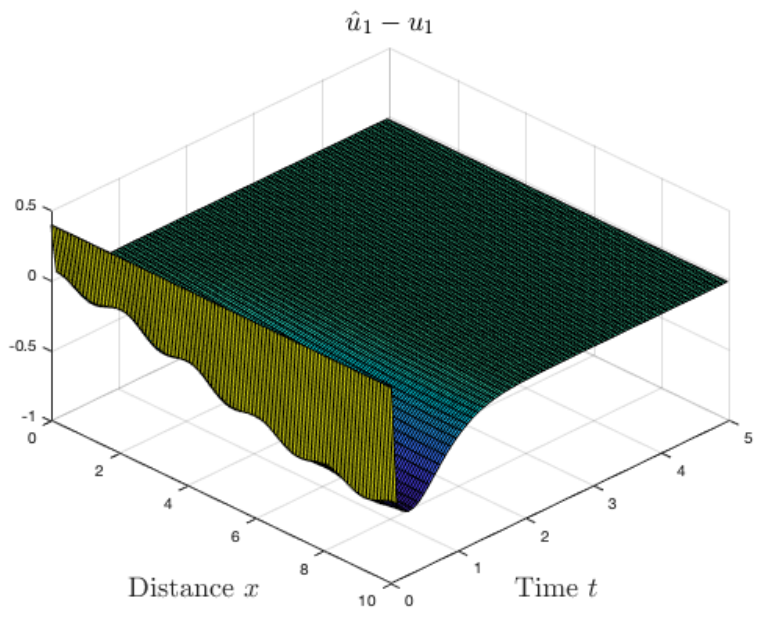

Fig. 2. Time and space evolution of the first estimation error

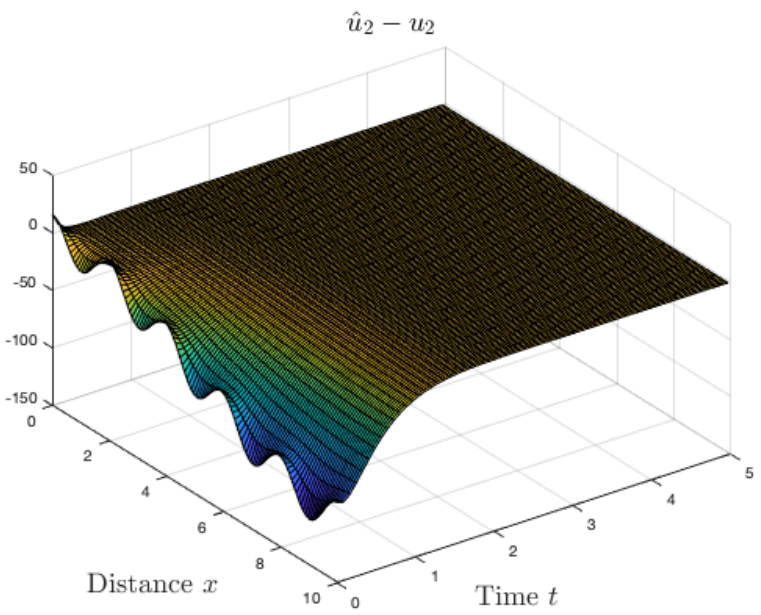

Fig. 3. Time and space evolution of the second estimation error

imposed by the parabolic operator, system is assumed to have stronger regularity and it is first mapped into a target system of PDEs. An observer for this system is designed, utilizing output correction terms and injection of output spatial derivatives. The extension of this methodology to more complex infinitedimensional systems with more states and different couplings will be subject for our future work.

\section{REFERENCES}

[1] F. Alabau-Boussouira, J.-M. Coron, and G. Olive, Internal controllability of first order quasilinear hyperbolic systems with a reduced number of controls, SIAM J. Control and Optimization, vol. 55, no. 1, pp. 300-323, 2017.

[2] A. A. Berryman, The origins and evolution of predator-prey theory, Ecology, vol. 73, pp. 1530-1535, 1992.

[3] G. Besançon, B. Pham, and D. Georges, Robust state estimation for a class of convection-diffusion-reaction systems, IFAC Workshop on Control of Systems Governed by partial Differential Equations, Paris, France, 2013.

[4] H. Bounit and H. Hammouri, Observer design for distributed parameter dissipative bilinear systems, Appl. Math. Comput. Sci., vol. 8, pp. 381$-402,1998$.
[5] D. Bourat and M. Saif, Nonlinear observer normal forms for some predator-prey models, IFAC Symposium on Nonlinear Control Systems, Toulouse, France, pp. 682-687, 2013.

[6] H. Brézis, Analyse Fonctionelle, Dunod, Paris, 1983.

[7] N. F. Britton, Reaction-diffusion equations and their applications to biology, Academic Press, New York, 1986.

[8] F. Castillo, E. Witrant, C. Prieur, and L. Dugard, Boundary observers for linear and quasi-linear hyperbolic systems with application to flow control, Automatica, vol. 49, no. 11, pp. 3180-3188, 2013.

[9] P. D. Christofides and P. Daoutidis, Feedback control of hyperbolic pde systems, AIChE Journal, vol. 42, no. 11, pp. 3063-3086, 1996.

[10] J.-M. Coron and P. Lissy, Local null controllability of the threedimensional Navier-Stokes system with a distributed control having two vanishing components, Invent. Math. 198, no. 3, pp. 833--880, 2014.

[11] E. Crépeau and C. Prieur, Approximate controllability of a reactiondiffusion system, Systems Control Lett., vol. 57(12), pp. 1048-1057, 2008.

[12] R. F. Curtain, Finite-dimensional compensator design for parabolic distributed systems with point sensors and boundary input, IEEE Transactions on Automatic Control, vol. 27, no. 1, pp. 98-104, 1982.

[13] M. Demetriou, Second order observers for second order distributed parameter systems, Syst. Control Lett., vol. 51, pp. 225-234, 2004.

[14] F. Di Meglio, M. Krstić, and R. Vasquez, A backstepping boundary observer for a class of linear first-order hyperbolic systems, European Control Conf., Zurich, Switzerland, pp. 1597-1602, 2013.

[15] L. C. Evans, Partial Differential Equations, American Mathematical Society, Graduate Studies in Mathematics, vol. 19, 1998.

[16] H. Hammouri, B. Targui, and F. Armanet, High gain observer based on a triangular structure, International Journal of Robust and Nonlinear Control, vol. 12(6), pp.497-518, 2002.

[17] A. Hasan, O. M. Aamo, and M. Krstić, Boundary observer design for hyperbolic PDE-ODE cascade systems, Automatica, vol. 68, pp.75-86, 2016.

[18] D. Henry, Geometric Theory of Semilinear Parabolic Equations, Lecture Notes in Mathematics, vol. 840, Springer-Verlag, Berlin, 1981.

[19] H. K. Khalil, High-gain observers in nonlinear feedback control, Advances in Design and Control. SIAM, 2017.

[20] C. Kitsos, G. Besançon, and C. Prieur, A high-gain observer for a class of 2x2 hyperbolic systems with $C^{1}$ exponential convergence, IFAC Workshop on Control of Systems Governed by Partial Differential Equations, Oaxaca, Mexico, 2019.

[21] C. Kitsos, G. Besançon, and C. Prieur, High-gain observer for $3 \times 3$ linear hyperbolic systems, submitted to Automatica, 2020.

[22] O. A. Ladyženskaja, V. A. Solonnikov and N. N. Uraltseva, Linear and quasilinear equations of parabolic type, Transl. of Math. Monographs 23, 1968.

[23] F. Mazenc and C. Prieur, Strict Lyapunov functions for semilinear parabolic partial differential equations, Mathematical Control and Related Fields, AIMS, vol. 1, pp. 231-250, 2011.

[24] T. Meurer, On the extended Luenberger-type observer for semilinear distributed-parameter systems, IEEE Trans. Autom. Control, vol. 58 (7), pp. 1732-1743, 2013.

[25] M. Pierre, Global existence in reaction-diffusion systems with control of mass: a survey, Milan J. Math, vol. 78(2), pp.417-455, 2010.

[26] K. Sakthivel, G. Devipriya, K. Balachandran, J. H. Kim, Controllability of a reaction-diffusion system describing predator-prey model, Numerical Functional Analysis and Optimization, vol. 31, pp. 831--851, 2010.

[27] A. Schaum, J. A. Moreno, J. Alvarez, and T. Meuer A simple observer scheme for a class of 1-D semi-linear parabolic distributed parameter systems, European Control Conf., Linz, Austria, pp. 49-54, 2015.

[28] A. Smyshlyaev and M. Krstić, Backstepping observers for a class of parabolic PDEs, Systems and Control Letters, vol. 54, pp. 613-625, 2005.

[29] H. Wang, F. Wang, and K. Xu, Modeling information diffusion in online social networks with partial differential equations, arXiv:1310.0505, 2013.

[30] A. V. Wouwer and M. Zeitz, Parameter estimation in distributed parameter systems, in Encyclopedia of Life Support Systems (EOLSS), H. Unbehauen, Ed. Oxford, U.K.: EOLSS Publishers, ch. Control Systems, Robotics and Automation, article No. 6.43.19.3, 2001.

[31] C. Xu, P. Ligarius, and J. P. Gauthier, An observer for infinitedimensional dissipative bilinear systems, Comput. Math. Appl., vol. 29, no. 7 , pp. $13-21,1995$. 